\title{
Backside TEM Sample Preparation With The Multi-Loader Flip-Stage
}

\author{
A. Buxbaum*, R. Schampers**, J. Bolt** \\ * FEI Company, 5350 NE Dawson Creek Drive, Hillsboro, OR 97124, U.S.A. \\ **FEI Company, Achtseweg Noord 5, Bldg 5651 GG Eindhoven, The Netherlands
}

The use of a DualBeam ${ }^{\mathrm{TM}}$ system (Focused Ion Beam and SEM columns on a single instrument) has established itself as the preferred method for TEM sample preparation for many applications. The ability to prepare TEM samples from a site specific region of interest (using the FIB) along with the built in capability of monitoring and inspection at each step of the process (using the SEM) makes it a uniquely powerful and flexible technique.

Along with the benefits of using a FIB for TEM sample preparation come challenges - some of which are due to the variation in milling properties of materials within the specimen. Samples having patterned structures within layers, such as contacts or vias in an IC device, can yield results with milling artifacts. The curtaining artifact [1] stems from a combination of different mill rates for different materials and masking during the mill process by structures in the films above the region of interest. This paper discusses the methodology and hardware employed for preparing TEM samples using an FEI Helios 400ML DualBeam ${ }^{\mathrm{TM}}$ and how some of the artifacts from FIB milling (namely curtaining over the region of interest) were avoided.

Semiconductor IC samples are particularly challenging for TEM sample preparation because of curtaining from the patterned thin film layers. Lamellae from these samples ( 1um thick) were lifted out and attached in-situ from the bulk stage to a molybdenum cartridge at the instruments flip stage. The molybdenum cartridges are specifically designed for automatic loading (tweezerless) into a DualBeam and a TEM, via Multi-Loader hardware. Within the DualBeam's flipstage, the cartridge can be tilted a full $360^{\circ}$ (alpha tilt, Fig. 1). This allows the user to attach the lamella to the cartridge in an inverted orientation (Fig. 2), such that the substrate of the IC device (bottom side) faces upward toward the ion column. This orientation, known as "Backside Milling", enables the thinning process to proceed with the focused ion beam incident on the smooth, non-patterned substrate and the region of interest just below (the gates of the device) is free from curtaining artifacts (Fig.3). In the TEM, the use of the cartridge allows for fast and precise sample loading along with lower drift and shorter settling times compared to $3 \mathrm{~mm}$ grids.

The flipstage design also accommodates a retractable STEM detector within the instrument. With the lamella at the milling orientation (facing the ion column), the thinning process can be monitored by real time imaging with the STEM detector using Bright Field (BF), Dark Field (DF) and High Angle Annular Dark Field (HAADF) modes - all simultaneously. When the thinning process is complete, the alpha tilt angle can be set for STEM imaging, such that the plain of the lamella is perpendicular to the electron beam (Fig. 4).

References

[1] Introduction to Focused Ion Beams, eds. L.A. Giannuzzi and F.A. Stevie, Springer (2005). 


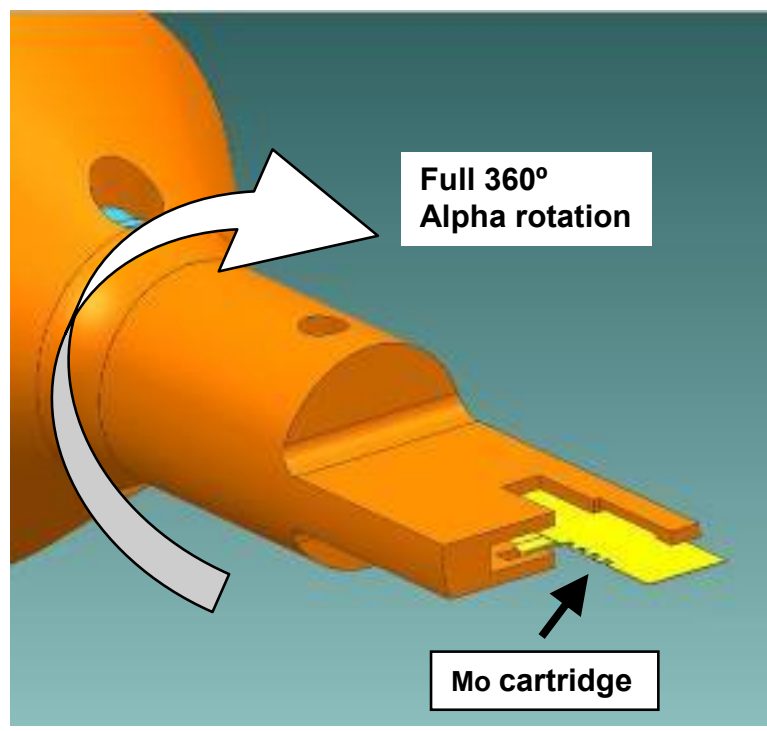

Fig. 1.

Schematic of the Multi-Loader Flipstage in the Helios 400ML DualBeam.

Lamellae are attached to molybdenum cartridges which are automatically transferred in and out of the instrument via a load-lock. The same cartridge is subsequently loaded into the TEM.

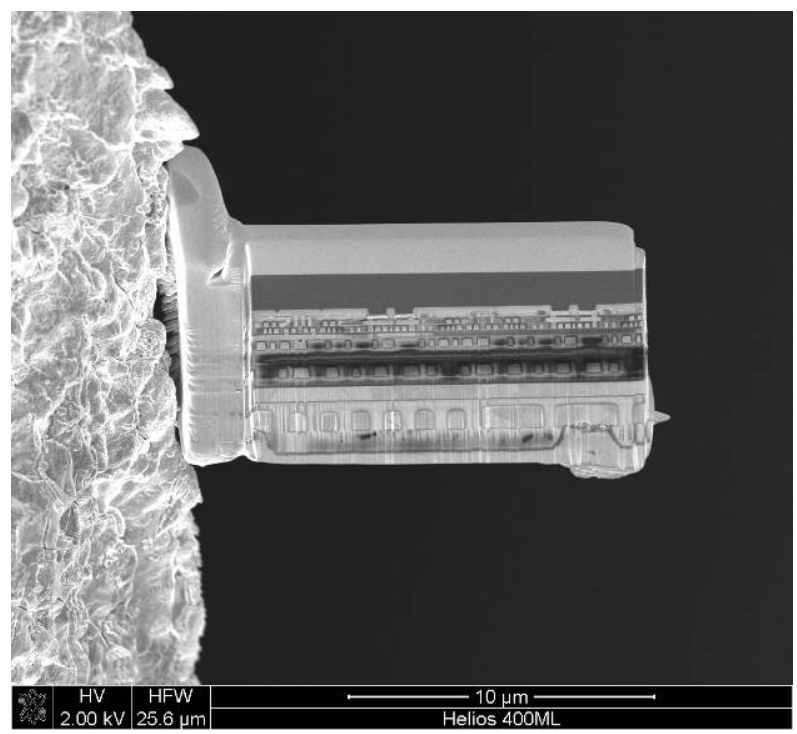

Fig. 3.

The backside milling orientation enables the thinning process to proceed with the FIB incident on the smooth substrate, thus mitigating the curtaining artifact from the region of interest.

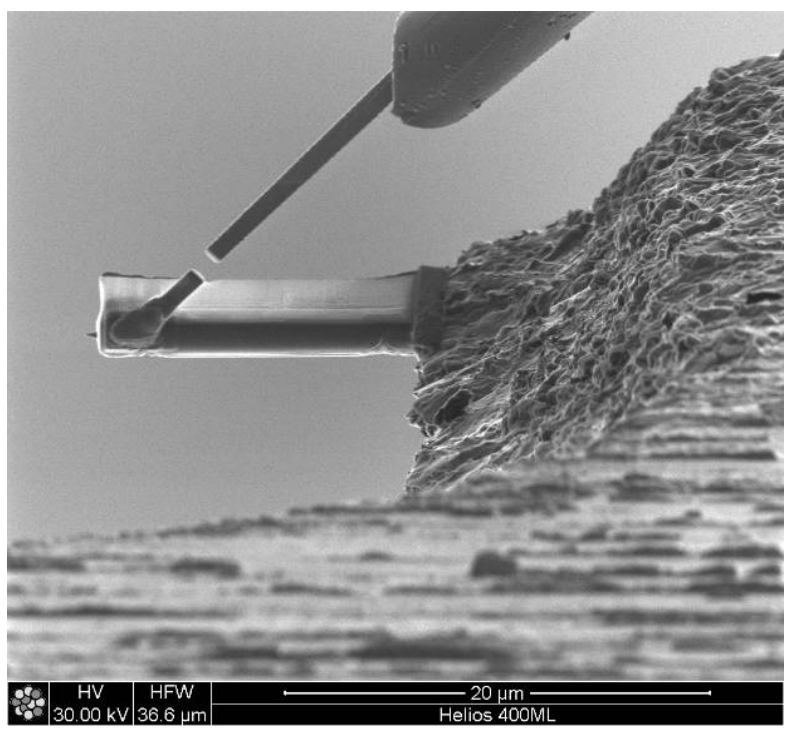

Fig 2.

Lamella is lifted out of the bulk sample and attached (shown) in-situ to the molybdenum cartridge. The unlimited alpha rotation allows to the user to attach the sample in a backside milling orientation: bottom/substrate side up (towards the ion beam).

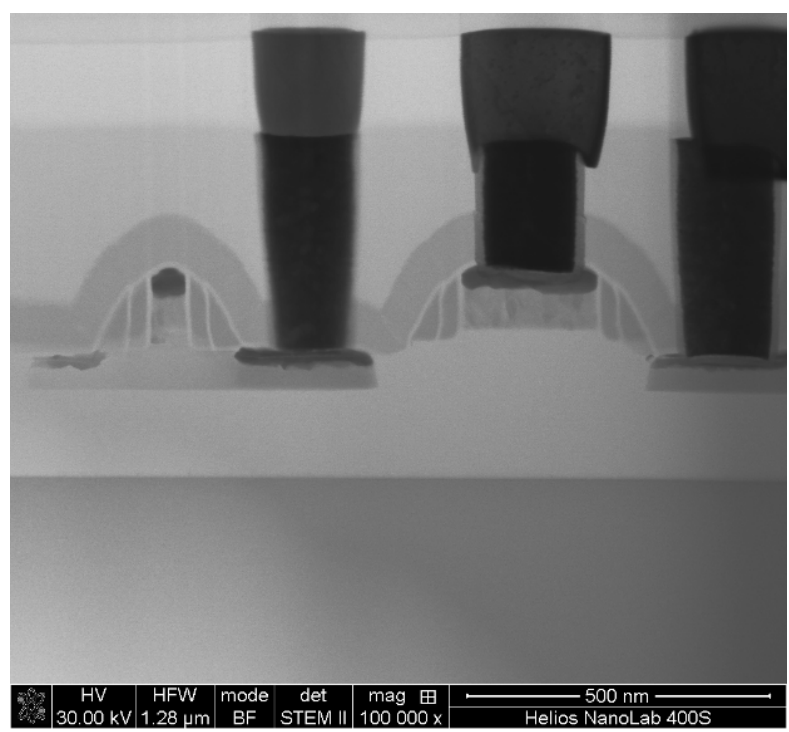

Fig 4.

A $30 \mathrm{keV}$ BF STEM image of the resulting TEM sample free from curtaining artifacts. 